\title{
Terrain referenced navigation using sand dunes and sand ripples in presence of tides
}

\author{
Ove Kent Hagen, Kjetil Bergh Ånonsen \\ Norwegian Defence Research Establishment (FFI) \\ P O Box 25, NO-2027 Kjeller, Norway \\ Email: ove-kent.hagen@ffi.no
}

\begin{abstract}
We investigated using terrain referenced navigation in shallow water in the presence of unknown tide, with an autonomous underwater vehicle equipped with a high resolution bathymetric sensor. The mission area had both variations, with large sand dunes, and flatness with small sand ripples. In order to deal with the unknown substantial tide and shallow water, we tested a non-linear estimator for horizontal position, tide level and sound speed errors, a four dimensional Rao-Blackwellized point mass filter. The algorithm received an initial position estimate from the inertial navigation system (INS), computed the probability density function for the position and environmental errors, tested for convergence, and provided a Gaussian approximation that could be used by the INS. The results showed that the algorithm converged consistently in both areas, more often in the sand dune area, but surprisingly with comparable to higher accuracy in the sand ripple area. This demonstrated that terrain referenced navigation worked as expected, on sand dunes, in shallow water with substantial tide. The flatness is sometimes only a matter of scale, and this technique should be considered used everywhere when equipped with high resolution sensors.
\end{abstract}

\section{INTRODUCTION}

Terrain referenced navigation is now an established technique for underwater navigation, and several systems exist for estimating vehicle position from a correlation of bathymetric measurements with a digital terrain model (DTM) of the sea floor. It is of course impossible for terrain referenced navigation to converge on a completely flat seafloor, and in those cases often complementary techniques are considered instead. This was recognized early for air craft applications, where it was suggested solved e.g. by augmenting the system with a scene matching system [1]. In underwater scenarios probably a range of techniques are required to solve this case [2].

In [3] the different factors influencing the performance of terrain referenced navigation are discussed, and in the experience of the authors, the flatness of the seafloor is sometimes simply a question of resolution. A seafloor that appears flat on a large scale, mapped by low resolution sensors, may contain detailed structures, revealed by high resolution sensors. In [4] pockmarks on the seafloor was effectively used for terrain referenced navigation in otherwise flat terrain, while in [5] unique finger prints from ice bergs scouring made terrain referenced navigation possible even in gently sloping terrain. Another known problem of terrain referenced navigation in conditions of small terrain variation is that an unknown tide may lead to a large bias in the position error if not properly accounted for [6]. It should also be noted that in [5] it was reported that low terrain variations may lead to false fixes

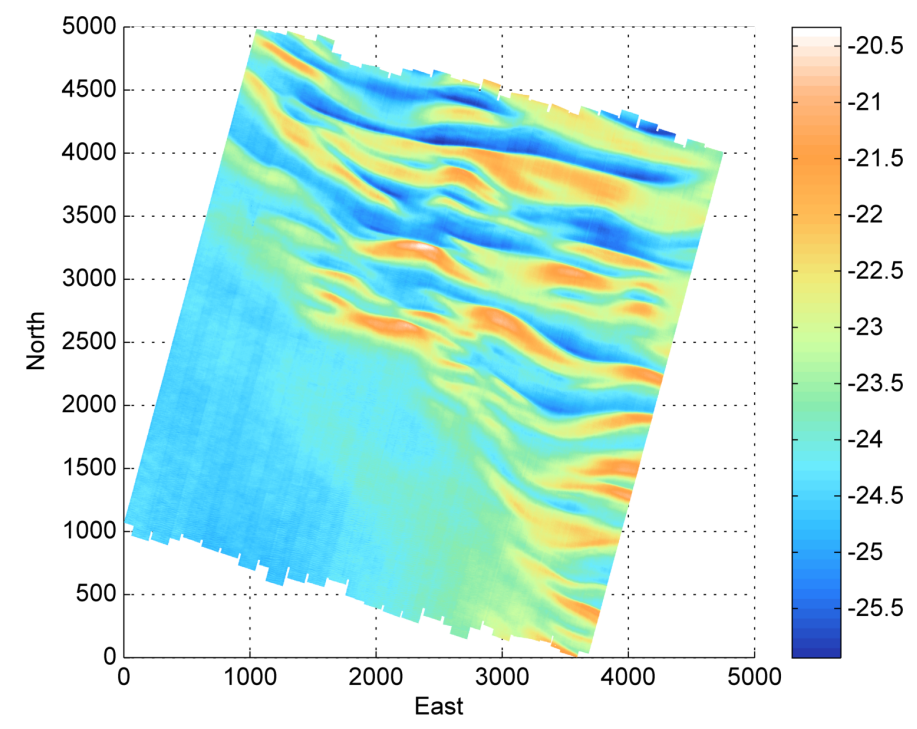

Fig. 1. The bathymetry outside Den Helder showing large regions with sand dunes.

when sensor noise occasionally correlate with map noise. This was suggested solved by an integrity system rejecting these false fixes. Later in [7], a robust estimator was introduced, which is able to handle this case without the need to reject the measurements.

In connection with general tests of the HUGIN autonomous underwater vehicle (AUV) in the shallow waters of the Netherlands, we were requested to test terrain referenced navigation. The bathymetry in the test area is mostly flat, but there also existed large regions with underwater sand dunes that could be used for terrain navigation, see Fig. 1. The sand dunes actually migrate slowly in time, which is a major challenge for terrain referenced navigation, but in the first test we were primarily interested in the performance using a recently constructed DTM from a surface ship. After we inspected the DTM, we became aware of small variations, sand ripples, in the flat area of the terrain, see Fig.2, and we decided to include this area in the test. Since the tide level in the area is rather high compared to the terrain variations, we used the terrain referenced navigation algorithm introduced in [8], which is able to concurrently estimate position, tide and sound speed errors.

The algorithm had previously only been tested in simulations, where it was found to robustly estimate all the parameters in both low- and high terrain variation scenarios. 


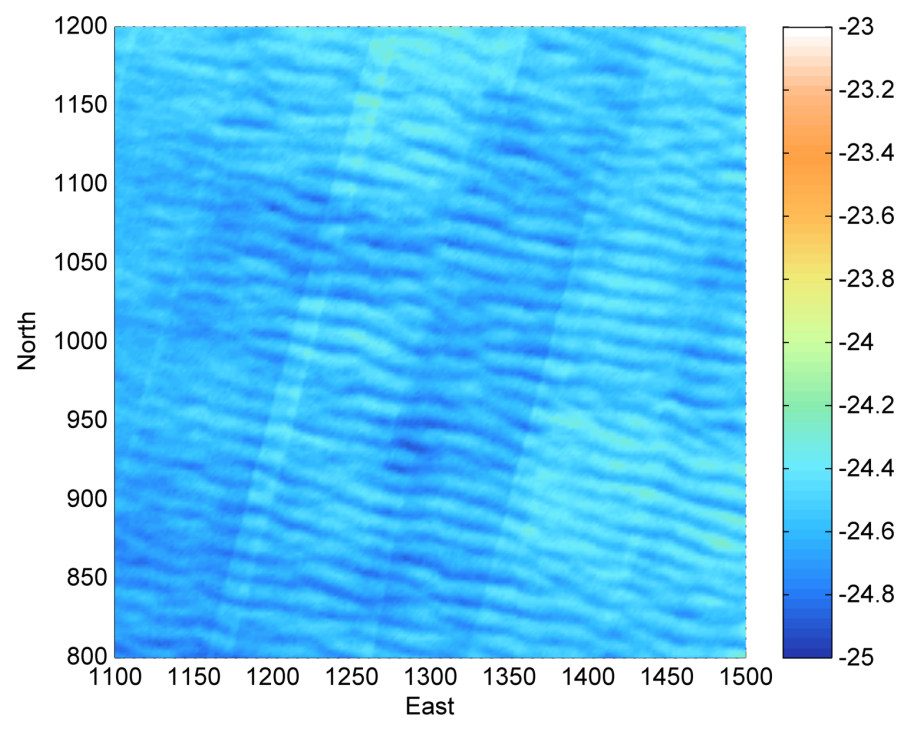

Fig. 2. The flat area actually has a large region with sand ripples.

This is, however, the first time the algorithm is tested on real data, and the first time we test any terrain referenced navigation algorithm on sand dunes and sand ripples.

\section{Method}

\section{A. Terrain referenced navigation}

Terrain referenced navigation has been used for decades in aircraft and missile navigation [9]-[11], and several approaches exist. We here concentrate on the so-called search area methods (see [12] for a classification of methods), which define a grid around the current, possibly uncertain position estimate, and try find the position within the search grid that gives the best match between the DTM and the terrain measurements. An early implementation of this concept was the TERCOM algorithm [9], which simply finds the position in the grid that minimizes the maximum absolute difference between the measured terrain profile and the DTM. A more sophisticated approach is to formulate the problem in a stochastic statespace model, and use a Bayesian estimator to estimate the probability density function (PDF) of the position based on the model and the measurements. As the measurement model is dependent on the DTM, which is often highly non-linear, non-linear Bayesian estimation methods are often required.

In [13] the point mass filter (PMF) algorithm, an approximation of the general Bayes filter equations using numerical quadrature on a grid [14], [15], was used for terrain referenced aircraft navigation. Another alternative is to use particle filters (PF), Monte Carlo based sampling methods [16], in which the sought PDF is estimated using a set of particles which are weighted and resampled in accordance with the measurements. Both of these alternatives have been used in terrain referenced navigation for AUVs [6], [17]-[19]. The PMF has been chosen in the HUGIN real-time terrain navigation system [5] because of its superior robustness properties when compared to PFs.

\section{B. Mathematical model}

Let $\boldsymbol{x}_{k}=\left(x_{k}, y_{k}, z_{k}\right)$ denote the AUV's position in a local north-east-down system at time $t=t_{k}$. A simplified dynamical model for the position states of the AUV is given by

$$
\boldsymbol{x}_{k+1}=\boldsymbol{x}_{k}+\boldsymbol{u}_{k}+\boldsymbol{v}_{k},
$$

where $\boldsymbol{u}_{k}$ is the integrated motion estimated by the inertial navigation system (INS) in the time interval $t_{k}$ to $t_{k+1}$, and $\boldsymbol{v}_{k}$ is a white stochastic process modeling the error drift in the INS estimate.

Let $\left(\boldsymbol{\xi}_{k}, \boldsymbol{\eta}_{k}, \boldsymbol{\zeta}_{k}\right)$ denote $m_{k}$ bathymetric depth measurements relative to the INS solution in a north-aligned and level body-system $N$, and let $h(\boldsymbol{x})=h(x, y)$ denote the terrain function, which gives the terrain depth as a function of global horizontal position. We introduce the measurement vector function $\boldsymbol{h}_{\boldsymbol{\xi}_{k}, \boldsymbol{\eta}_{k}}\left(\boldsymbol{x}_{k}\right)=\left\{h_{i}\right\}_{i=1}^{m_{k}}$ with scalar components defined by

$$
h_{i}=h\left(x_{k}+\xi_{k, i}, y_{k}+\eta_{k, i}\right)-z_{k} .
$$

In [8] it was shown that a measurement model with a linear tide and sound speed error model for a swath bathymeter, in low terrain variations, is given by

$$
\boldsymbol{\zeta}_{k}=\boldsymbol{h}_{\boldsymbol{\xi}_{k}, \boldsymbol{\eta}_{k}}\left(\boldsymbol{x}_{k}\right)+\boldsymbol{H}_{k} \boldsymbol{\epsilon}_{k}+\boldsymbol{w}_{k} .
$$

Here we have introduced the environment error state vector $\boldsymbol{\epsilon}_{k}=[\delta z, \delta c]_{k}^{T}$ for the tide error $\delta z$ and the sound speed error $\delta c$, and $\boldsymbol{w}_{k}$ is a white stochastic process modeling the sensor and DTM errors. The $m_{k} \times 2$ matrix $\boldsymbol{H}_{k}=\left\{H_{i, k}\right\}$, where

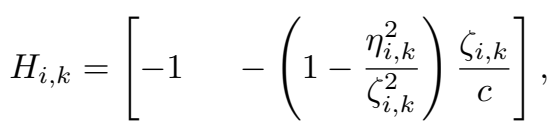

projects the environment errors onto a corresponding depth error, where $c$ is the currently known sound speed.

\section{Rao-Blackwellized point mass filter}

We will give a short description of the Rao-Blackwellized PMF (RB-PMF) introduced in [8], by first looking at the regular PMF formulation. The search grid is defined as a 2D bounded domain $G_{k}$ in the earth tangent plane, enclosing the current INS solution. The error of the INS is denoted as $\delta \boldsymbol{x}_{k}$, a position vector in $G_{k}$, and $Z_{k}$ denotes the set of all measurements between the times $t_{1}$ and $t_{k}$. Our goal is to estimate the position error filter PDF $p\left(\delta \boldsymbol{x}_{k} \mid Z_{k}\right)$, conditioned on all the measurements so far in what we call the correlation period $\left[t_{0}, t_{k}\right]$. Since $\delta \boldsymbol{x}_{k}$ is a Markov process, its PDF evolves in time according to the convolution integral [12]

$$
p\left(\delta \boldsymbol{x}_{k+1} \mid Z_{k}\right)=\int_{G_{k}} p_{v_{k}}\left(\delta \boldsymbol{x}_{k+1}-\delta \boldsymbol{x}_{k}\right) p\left(\delta \boldsymbol{x}_{k} \mid Z_{k}\right) d \delta \boldsymbol{x}_{k},
$$

where $p_{v_{k}}(\cdot)=p\left(\delta \boldsymbol{x}_{k+1} \mid \delta \boldsymbol{x}_{k}\right)$ is the Markovian transition kernel for the error drift.

Bayes' theorem provides the mechanism for the measurement update

$$
p\left(\delta \boldsymbol{x}_{k} \mid Z_{k}\right)=\frac{p_{w_{k}}\left(\boldsymbol{\zeta}_{k}-\boldsymbol{h}_{\boldsymbol{\xi}_{k}, \boldsymbol{\eta}_{k}}\left(\boldsymbol{x}_{k}+\delta \boldsymbol{x}_{k}\right)\right) p\left(\delta \boldsymbol{x}_{k} \mid Z_{k}\right)}{\alpha_{k}},
$$

where $\alpha_{k}=\int_{G_{k}} p_{w_{k}}\left(\boldsymbol{\zeta}_{k}-\boldsymbol{h}_{\boldsymbol{\xi}_{k}, \boldsymbol{\eta}_{k}}\left(\boldsymbol{x}_{k}+\delta \boldsymbol{x}_{k}\right)\right) p\left(\delta \boldsymbol{x}_{k} \mid Z_{k}\right) d \delta \boldsymbol{x}_{k}$ is a normalization factor, and $p_{w_{k}}(\cdot)$ denotes the PDF for the sensor measurement error. 
Together, the equations (5) and (6) form the recursive Bayesian estimator equations for terrain referenced navigation. The recursion is started by a known initial PDF $p\left(\delta \boldsymbol{x}_{0} \mid Z_{0}\right)=$ $p_{0}\left(\delta \boldsymbol{x}_{0}\right)$ at $t_{0}$ that depends on the initial INS accuracy, and the filter then runs recursively for each ping until a convergence or divergence criterion is met at $t_{K}$. The PMF approximation is found by representing the filter PDF in (5) and (6) by point masses on a regularly spaced grid representation of $G_{k}$, and by calculating the integrals through straightforward quadrature. Once the PMF approximation of the PDF is found, all requested statistics of the INS position error can be calculated directly from the approximated PDF [12].

Now, an optimal estimator including tide and sound speed error, could simply augment the state vector above with the states $\epsilon_{k}$, but this would require a 4D PMF estimator which is computationally demanding, see e.g. [19] that used a 3D estimator. If we assume the tide and sound speed error is constant in the correlation period, i.e $\boldsymbol{\epsilon}_{k+1}=\boldsymbol{\epsilon}_{k}$, both the dynamic equation and measurement model is linear in the sound speed and depth error subspace that contains $\epsilon_{k}$. Since the noise is also Gaussian, the problem can be reformulated to a more efficient estimator, by applying Rao-Blackwell's and Bayes' theorem [20] for the joint PDF for the position and environmental errors. First we note that the joint PDF can we written as

$$
p\left(\delta \boldsymbol{x}_{k}, \boldsymbol{\epsilon}_{k} \mid Z_{k}\right)=p\left(\delta \boldsymbol{x}_{k} \mid Z_{k}\right) p\left(\boldsymbol{\epsilon}_{k} \mid \delta \boldsymbol{x}_{k}, Z_{k}\right) .
$$

The first PDF factor is found using the PMF estimator above, and the second PDF factor can be optimally found using Kalman filters [21], conditioned on each grid point of the PMF. Since the linear model is not dependent on the particular grid point, a common Kalman gain can be used, and the estimated covariance matrix $\boldsymbol{P}_{\boldsymbol{\epsilon}_{k}}$ from the Kalman filter is also common for all grid points.

In order to use the results of the PMF, we first need to make sure that there is enough information coming from the seafloor and the measurements into the filter, such that the estimates are not correlated with the initial INS solution. This is done by checking the Kullback-Leibler divergence from the initial PDF [22]. If we do have a candidate solution PDF, we then need to check if it is close enough to a Gaussian in order to use this in the Kalman filter of the INS. This is done by checking the Hellinger distance between the candidate PDF and a Gaussian approximation of the same PDF [22].

\section{RESULTS AND DISCUSSION}

\section{A. Experiment}

In January 2018 the Norwegian Defence Research Establishment (FFI) conducted a series of experiments with the HUGIN HUS AUV (see Fig. 3) in collaboration with the Dutch Defence Materiel Organisation (DMO) and the Netherlands Organisation for applied scientific research (TNO). The overall goal of the experiments was to test state-of-the-art AUV technology in the Dutch coastal water environment. One of the tests was to evaluate terrain referenced navigation in areas of small terrain variations in combination with tides and shallow water. For this purpose, days before the mission, a reference DTM of one meter

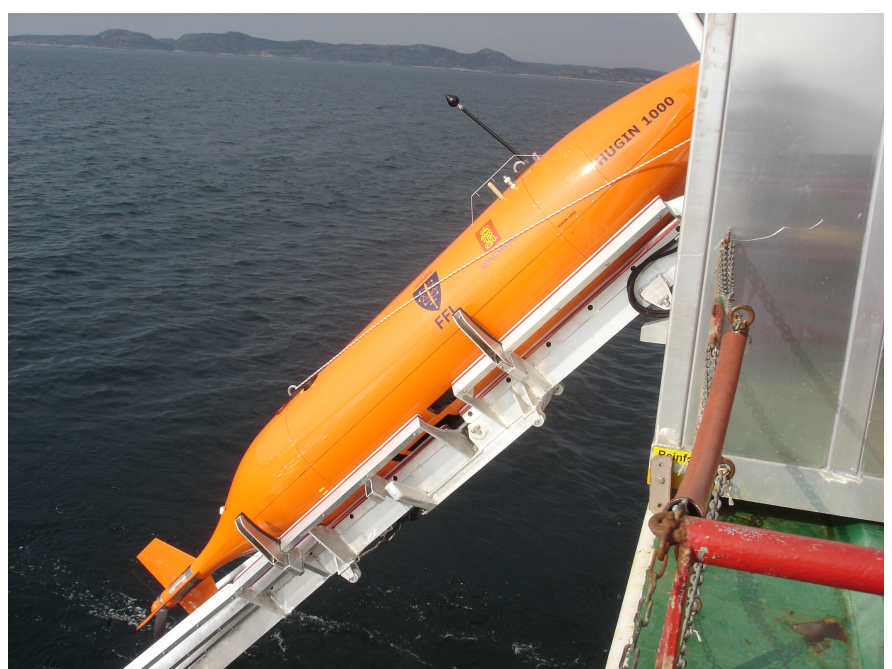

Fig. 3. The HUGIN 1000 HUS seconds before launch from FFI's research vessel H.U. Sverdrup II.

horizontal resolution was surveyed by the Dutch naval surface ship HNLMS Luymes in the lowest astronomical tide (LAT) datum. Part of the DTM has wide area structures of sand dunes with a typical height of 4-5 meters, as seen in the northern and eastern part in Fig.1. The south-western part is essentially flat, and initially we assumed that it was not possible to converge with terrain referenced navigation in this area. After closer inspection of the DTM, we found structures of small sand ripples with a typical height of 10-30 centimeters. A mission of the HUGIN HUS that visited both these areas was selected for the experiment. Only the EM 2040 multibeam echosounder (MBE) on board HUGIN HUS was used, and because of the shallow water, the AUV could not increase footprint coverage by increasing its altitude much, which is a typical tactic to use in these scenarios. Still, in this case, the typical swath width of the MBE was about 40 - 50 meters. In addition to the DTM, the surveyors of the HNLMS Luymes also provided us with tidal predictions and measurements in the mission area in the LAT datum.

The RB-PMF was run offline with a search grid of $200 \mathrm{x}$ 200 meters, with 1 meter resolution. The HUGIN HUS INS solution was used as input navigation, but a uniform initial PDF was used in the filter, and no tidal information was used in the filter other than a knowledge of the general level of tide in the area. This was chosen in order to study more directly the performance of the RB-PMF. The convergence criterion of the RB-PMF was kept fixed for both areas, in order to compare the relative performance between the two areas.

A ground truth was constructed by post-processing the navigation data using NavLab [23]. The accuracy was somewhat limited since we only had GPS at the start and at the end of the mission, but can in some degree be used to test consistency of the terrain referenced navigation estimates. The provided measurements of the tide can also be used as a reference to the evaluation of the estimated tide from terrain referenced navigation. The number of converged fixes per meters traveled will indicate how often the INS will receive an update. 


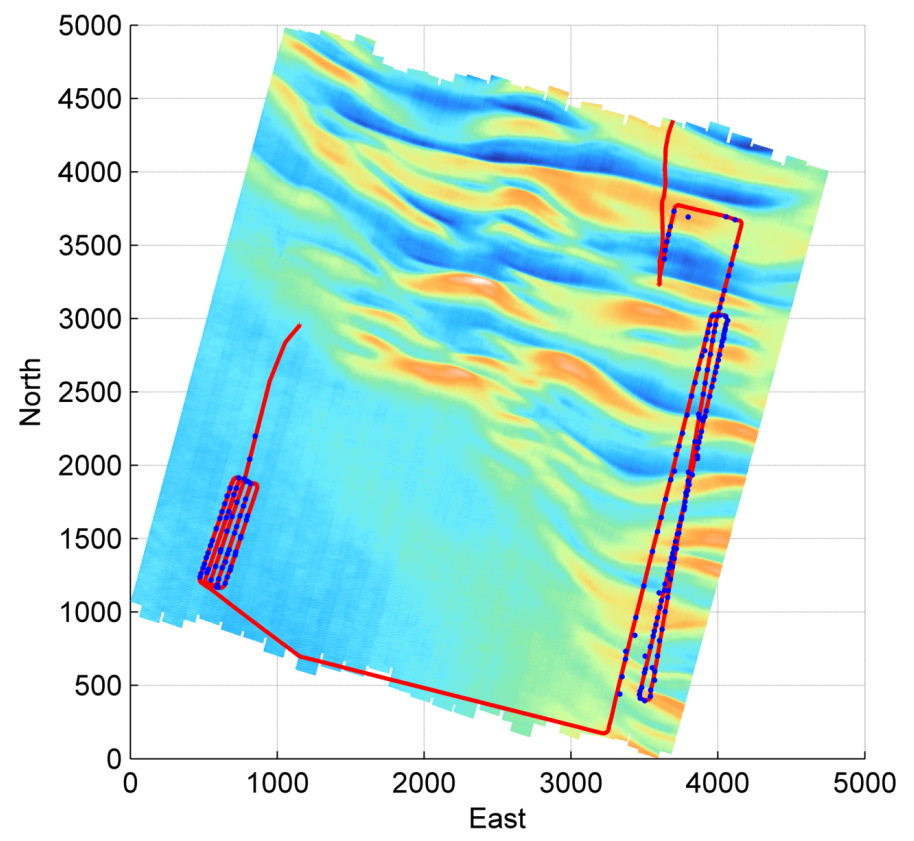

Fig. 4. Mission results overview. Red curve is the post-processed ground truth trajectory of the AUV. Blue dots are converged position estimates from terrain navigation.

\section{B. Flat area}

The AUV is going back and forth in the flat area for a total length of about 8 kilometers, doing sonar range tests on mine objects. Fig. 4 shows that the algorithm converges 57 times in this area, i.e. 7.1 fixes per kilometer. See also Fig.5, which shows the difference between the terrain referenced navigation estimates and the post-processed ground truth navigation, along with the combined standard deviations of the ground truth and terrain referenced navigation solutions. The position estimates have negligible bias compared to the ground truth, and with a combined standard deviation of about 2.4 meters in north-south and about 5.8 meters in east-west. The estimates are mostly consistent, except for an isolated case of gross error. The mean self reported accuracy of the RB-PMF is 2.1 meters in north-south and 3.2 meters in east-west. The large offset in the depth estimate is essentially the algorithm's estimate of the combined offset in depth due to uncompensated tide, atmospheric pressure and geopotential anomaly, in the ground truth navigation.

\section{Wave area}

In the wave area the AUV is also mostly traveling back and forth as part of the original terrain referenced navigation test, but this time along longer lines, for a total of 10 kilometers, see Fig.4. In this area the algorithm converges 134 times, i.e. 13.4 fixes per kilometer. In Fig.6 the difference between the converged estimates and the ground truth reference shows consistent performance except for a singular fix. The accuracy is periodically lower in the eastern direction, but has a negligible bias, and is otherwise fair in general with a combined standard deviation of about 9.3 meters. The northern direction accuracy is consistently good, but with a bias of nearly -3 meters, and a combined standard deviation of 5.0 meters. The bias is not constant, it is larger in the start than at the end, where it is
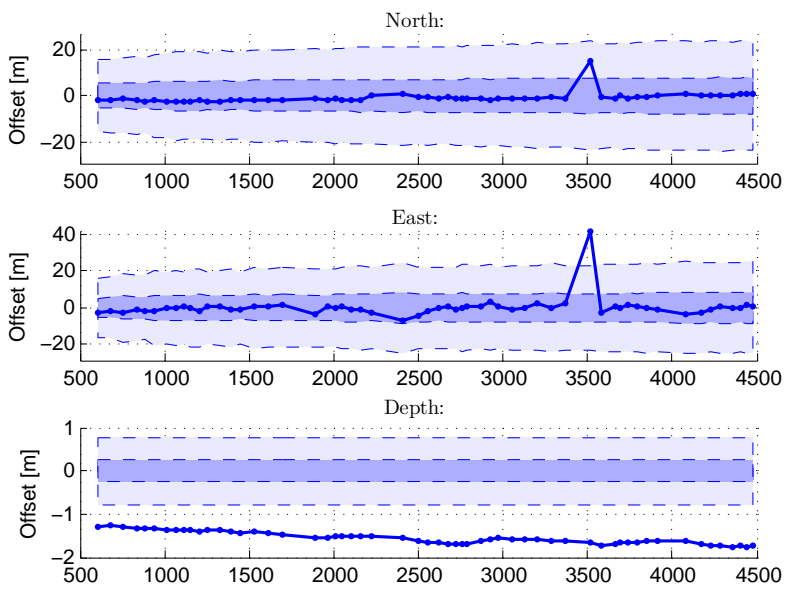

Fig. 5. Difference between ground truth and terrain referenced navigation (blue) in the flat area. The blue areas shows the combined one and three sigma confidence intervals.
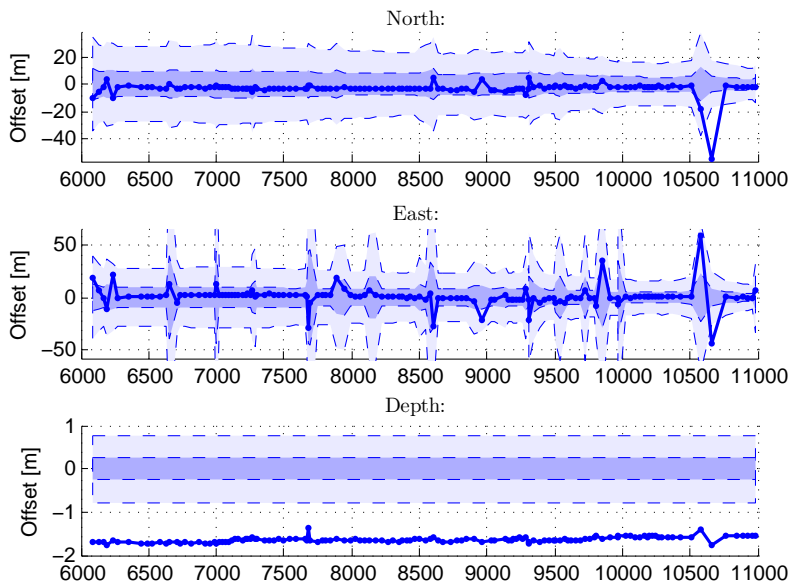

Fig. 6. Difference between ground truth and terrain referenced navigation (blue) in the wave area. The blue areas shows the combined one and three sigma confidence intervals.

about -2 meters. In the wave area the reported mean standard deviation of the RB-PMF is 2.0 meters in north-south and 6.0 meters in east-west. The level of the depth offset is also consistent with the one found in the flat area. If we compensate for atmospheric pressure, we can also make a fair comparison with the measured tide level. The absolute pressure sensor of HUGIN HUS shows an equivalent water level of about 15 centimeters from measurements on the ship deck of H.U. Sverdrup II before the mission. Using this compensation, all the RB-PMF estimates of tide are compared with the tide measurements provided by HNLMS Luymes in Fig.7.

\section{Discussion}

The results of section III-B and III-C show that the RBPMF converges, and in general with good accuracy, in both the flat area and the wave area. The terrain referenced navigation solution was found to be mostly consistent with the ground 


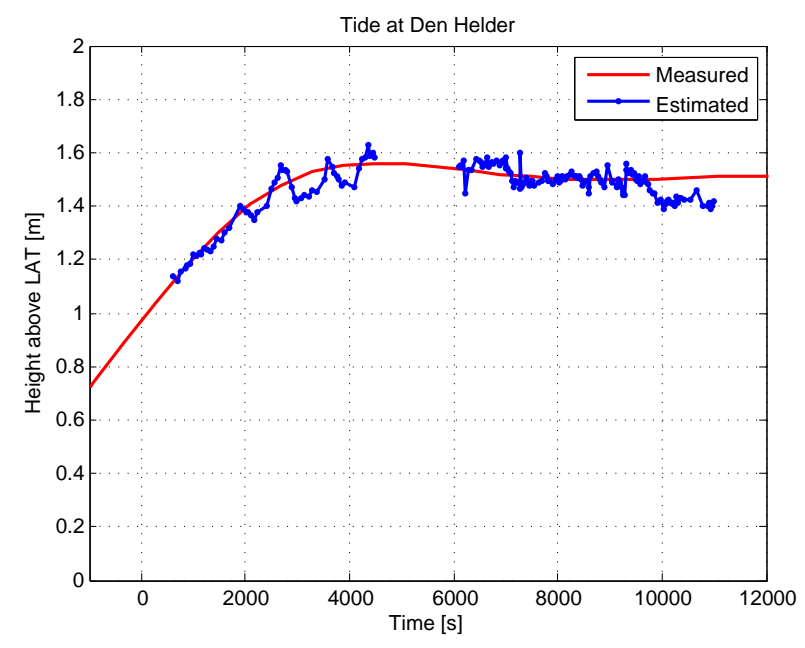

Fig. 7. Comparison of tide measurements (red) and estimated tide from HUGIN HUS using terrain referenced navigation (blue).

truth reference. In the wave area there are periods of lower estimated accuracy from the RB-PMF in the eastern direction. There is also a bias between the PB-PMF and the ground truth in the northern direction in the same area. In both areas the RB-PMF's tide estimation was mostly in good agreement with the measured tide. As expected, the algorithm converges more often in the wave area, but most surprisingly we often get convergence in the flat area, and the accuracy there is overall more accurate. We have seen this happen on larger scale features in earlier tests, such as ice berg scourings [5] and pockmarks [4], and by picking up some terrain signals in the outer beams of wide swath interferometers at low altitude [22]. In [24] and [25] terrain navigation was also found to converge on high resolution data, using data from previous mission lines, for a mix of small and large features in the Barents Sea. But we have never seen reports on convergence on such a large scale on a seemingly flat seafloor.

The sand ripples in the flat part establish a unique spatial signature, that can be measured with high resolution echo sounders. These were only possible to use for navigation since both HNLMS Luymes and HUGIN HUS carries such sensors, and because of the shallow water enabling the generation of a high resolution DTM from a surface ship as well. The periodic increase in eastern direction in the wave area is probably due to the wave direction of the sand dunes, as the fronts are mostly aligned in the eastern direction, and terrain referenced navigation gets more information in the wave propagation direction, which is mostly north. This probably explains why the accuracy reported by the RB-PMF is better in the eastern direction in the flat part. The northern bias in the comparison in the wave area may be explained by an error in the ground truth itself. As noted the ground truth position was found through post processing the INS data with only GPS fixes for position update, only at the start and at the end of the dive. There will in this case be substantial drift in the INS that can not be completely compensated for by post processing, and the accuracy will be best near the GPS fixes, which is what we observe in the comparison with the RB-PMF. The residual bias is then comparable to the accuracy of the GPS. In any case the RB-PMF estimates was found to be overall consistent with the ground truth.

The sand ripples are probably volatile features, so it is questionable if this can be used for globally referenced terrain navigation on older DTMs. However, this test shows that they can be used in-mission, for simultaneous localization and mapping using terrain navigation, and with recently constructed DTMs e.g.from earlier missions. In the wave area, it is known that the sand dunes migrate slowly, and older DTMs should be possible to use, at least with some adjustments to the current algorithm, allowing for such motion.

\section{CONCLUSIONS}

We have demonstrated the use of AUV terrain referenced navigation using sand dunes and sand ripples in shallow water, in the case of using a recently surveyed high resolution DTM and a high resolution $\mathrm{MBE}$.

The RB-PMF algorithm converged frequently in the sand dune area, and surprisingly even though not as often, also in the flat seafloor area, because of the sand ripples found there. Most surprisingly, the accuracy reported by the RB-PMF was in general better in the flat area, but this is probably due to the AUV encountering periodically flat and featureless areas within the sand dune wave pattern. The estimated tide from the algorithm was found to be in agreement with the measured tide for both areas. The estimates were found to be consistent with the exception of only two cases of gross errors. A bias found between the RB-PMF and the post-processed INS solution was found to be within the expected combined performance of the two solutions.

The findings herein shows that one should reconsider the use of terrain referenced navigation even in the case of seemingly flat seafloor. The introduction of high resolution sensors have a great impact on the information that can be perceived from any seafloor. If we can pick up that information in both the sensor and the DTM, terrain referenced navigation can be used out-of-the-box.

In this sense flatness is often only a matter of scale. But the small features then used may be volatile, so at some point we probably cannot use old DTMs for this purpose. However, in simultaneous localization and mapping scenarios, where the DTM is created in mission by the AUV itself, we could exploit this.

It would be interesting to see if there is an overall performance difference between the two areas after integrating the RB-PMF with the INS, but this may require the construction of a better ground truth solution, which again may be difficult for this particular data set.

\section{ACKNOWLEDGMENT}

The authors thank the Dutch MOD for granting the possibility of performing this experiment, and the crew and surveyors of the HNLMS Luymes for providing the DTM and tide data. We also thank the crew and HUGIN operators of the H.U. Sverdrup II for performing the experiment, and especially Petter Lågstad for all his help in gathering the data. 


\section{REFERENCES}

[1] A. Henley, "Terrain aided navigation: Current status, techniques for flat terrain and reference data requirements," in Proceedings of the IEEE Position Location and Navigation Symposium (PLANS), Las Vegas, NV, Mar. 1990, pp. 608-615.

[2] P. E. Hagen, Ø. Hegrenæs, B. Jalving, Ø. Midtgaard, M. Wiig, and O. K Hagen, Underwater Vehicles. Vienna, Austria: In-Tech Education and Publishing, 2009, ch. Making AUVs Truly Autonomous, pp. 129-152.

[3] O. K. Hagen and K. B. Anonsen, "On the performance of terrain navigation," in UDT Europe 2016. UDT, 2016.

[4] K. B. Ånonsen and O. K. Hagen, "Terrain aided underwater navigation using pockmarks," in Proceedings of the MTS/IEEE OCEANS 2009 Conference, Biloxi, MS, October 2009.

[5] O. K. Hagen, K. B. Ånonsen, and M. Mandt, "The HUGIN real-time terrain navigation system," in Proceedings of the MTS/IEEE OCEANS 2010 Conference, Seattle, WA, USA, 2010.

[6] G. T. Donovan, "Position error correction for an autonomous underwater vehicle inertial navigation system (INS) using a particle filter," IEEE Journal of Oceanic Engineering, vol. 37, no. 3, pp. 431-445, 2012.

[7] S. Dektor and S. Rock, "Robust adaptive terrain-relative navigation," in Proceedings of MTS/IEEE OCEANS 2014 Conference, St. John's, NL, Canada, 2014.

[8] O. K. Hagen and K. B. Ånonsen, "Improving terrain navigation by concurrent tidal and sound speed error estimation," in Proceedings of MTS/IEEE OCEANS 2013 Conference, San Diego, CA, USA, 2013.

[9] J. Golden, "Terrain contour matching (TERCOM): A cruise missile guidance aid," in Image Processing for Missile Guidance, T. Wiener, Ed., vol. 238. San Diego, CA: The Society of Photo-Optical Instrumentation Engineers, 1980, pp. 10-18.

[10] D. Boozer and J. Fellerhoff, "Terrain-aided navigation test results in the AFTI/F-16 aircraft," Journal of The Institute of Navigation, vol. 35, no. 2, pp. 161-175, 1988 .

[11] J. Hollowell, "Heli/SITAN: A terrain referenced navigation algorithm for helicopters," in Proceedings of the IEEE Position Location and Navigation Symposium (PLANS), Las Vegas, NV, Mar. 1990, pp. 616625.

[12] K. B. Ånonsen, "Advances in terrain aided navigation for underwater vehicles," Ph.D. dissertation, Norwegian University of Science and Technology, 2010.

[13] N. Bergman, L. Ljung, and F. Gustafsson, "Terrain navigation using Bayesian statistics," IEEE Control Systems Magazine, vol. 19, no. 3 , pp. 33-40, Jun. 1999.
[14] R. Bucy, "Bayes theorem and digital realizations for non-linear filters," The Journal of the Astronautical Sciences, vol. XVII, no. 2, pp. 80-94, Sep.-Oct. 1969.

[15] R. Bucy and K. Senne, "Digital synthesis of non-linear filters," Automatica, vol. 7, no. 3, pp. 315-322, May 1971.

[16] A. Doucet, S. Godsill, and C. Andrieu, "On sequential monte carlo sampling methods for Bayesian filtering," Statistics and Computing, vol. 10, no. 3, pp. 197-208, 2000.

[17] R. Karlsson, F. Gustafsson, and T. Karlsson, "Particle filtering and Cramer-Rao lower bound for underwater navigation," in Proceedings IEEE Conference on Acoustics, Speech and Signal Processing, Hong Kong, April 2003.

[18] K. B. Ånonsen, O. Hallingstad, O. Hagen, and M. Mandt, "Terrain aided AUV navigation - a comparison of the point mass filter and terrain contour matching algorithms," in Proceedings of the Undersea Defence Technology Conference (UDT) Europe 2005, Amsterdam, the Netherlands, June 2005.

[19] K. Ånonsen and O. Hallingstad, "Terrain aided underwater navigation using point mass and particle filters," in Proceedings of the IEEE/ION Position Location and Navigation Symposium (PLANS) 2006, San Diego, CA, April 2006.

[20] G. Casella and P. Robert, "Rao-blackwellisation of sampling schemes," Biometrika, vol. 83, no. 1, p. 81..94, 1996.

[21] A. Gelb, Ed., Applied Optimal Estimation. Cambridge, MA: The MIT Press, 1974

[22] O. K. Hagen, K. B. Ånonsen, and T. O. Sæbø, "Low altitude AUV terrain navigation using an interferometric sidescan sonar," in Proceedings of the MTS/IEEE OCEANS 2011 Conference, Kona, HI, USA, 2011.

[23] K. Gade, "NavLab, a generic simulation and post-processing tool for navigation," European Journal of Navigation, vol. 2, no. 4, pp. 51-59, November 2004

[24] O. K. Hagen, K. B. Ånonsen, and T. O. Sæbø, "Toward autonomous mapping with AUVs - Line-to-line terrain navigation," in Proceedings of the MTS/IEEE OCEANS 2015 Conference, Washington, DC, USA, 2015.

[25] K. B. Ånonsen, O. K. Hagen, and E. Berglund, "Autonomous mapping with AUVs using relative terrain navigation," in Proceedings of the MTS/IEEE OCEANS 2017 Conference, Anchorage, AK, USA, 2017. 